\title{
TRANSVERSE COUPLING IMPEDANCE OF THE VEPP-4M COLLIDER: MEASUREMENTS AND SIMULATIONS
}

\author{
V.Kiselev, V.Smaluk, V.Zorin, BINP, Novosibirsk, Russia
}

\begin{abstract}
The main contribution into the total impedance of the VEPP-4M is made by places of non-uniformity of vacuum chamber, such as sharp change of cross section, ceramic inserts, vertical electrostatic separators and by HOMs of cavities. Computer code GdfidL is used for the estimate of impedances in some of above structures. A new method for measurement of transverse coupling impedance has been used. The method is based on measuring of closed orbit deviation caused by localized impedance. Transverse impedance acts on the beam as a defocusing quadrupole, strength of which depends on the beam current. If a local bump of closed orbit has been created at the impedance location, then the orbit deviation occurs while varying the beam current. The local impedance can be evaluated using the orbit deviation measured. The results of the measurements and numerical calculations are presented and compared.
\end{abstract}

\section{INTRODUCTION}

There are some precise methods to measure integral characteristics of the ring transverse impedance. So, the resistive component of the transverse impedance can be found by measurements of coherent betatron oscillations decrement of fast damping. Calculated using such measurements, resistive component of the VEPP-4M transverse impedance is $\operatorname{Re} Z_{\perp, 1} \approx 0.6 \mathrm{M} \Omega / \mathrm{m}$.[1] The reactive component of transverse impedance can be found by measuring of current dependence of coherent betatron frequency shift. Evaluated from this measurements, integral beta-weighted value of reactive transverse impedance is $<\operatorname{Im} Z_{\perp, 1} \cdot \beta_{y}>\approx 22 \mathrm{M} \Omega$, the respectively $\operatorname{Im} Z_{\perp, 1} \approx 2.0 \mathrm{M} \Omega / \mathrm{m}$. For the radial direction these values are 5 times less.

Measurement of the impedance of vacuum chamber local elements is rather difficult. Method for measurement of local transverse impedance using beam orbit measurement system is developed and successfully used in CERN [2]. The variation with the current of the betatron phase advance between two points in the ring can be used to estimate the imaginary part of the transverse impedance located between those two points:

$$
\Delta \Psi=\frac{\Delta I_{a} \cdot \sum_{k} \beta_{\perp k} \cdot \int \operatorname{Im}\left\{Z_{\perp k, 1}\left(c \xi / \sigma_{s}\right)\right\} \cdot \mid \tilde{\rho}\left(c \xi / \sigma_{s}\right)^{2} \cdot d \xi}{2 \sqrt{2 \pi} \cdot(E / e)} .
$$

Here $\Delta \Psi$ is betatron phase advance from longitudinal positions $s_{0}$ to $s_{1}$, and $\sum_{k}$ is a sum over all impedance sources in that interval with dipole component of the transverse impedance $Z_{\perp k, 1}$. Amplitude value of the beam current defined as $I_{a}=\bar{I} \cdot P / \sqrt{2 \pi} \sigma_{s}$, where $\bar{I}$ is average current, $P$ is circumference, $\sigma_{s}$ is the RMS bunch length. Integral value of the arc sections transverse impedance of the LEP ring measured by this method is $69 \mathrm{M} \Omega$. These measurements were possible because of comparatively large value of coherent phase advance for the LEP ( $\left.\Delta \Psi_{\text {max }} \approx 0.65\right)$. For the VEPP-4M $\Delta \Psi_{\max } \approx 0.12$ and impedance distribution is more complicated, so we decided to try another method of local impedance measurement [3], also using beam orbit system, but in integral regime, when accuracy of measurements is greater by order because of multiple data averaging.

\section{BASIS OF BUMP METHOD AND MEASUREMENT TECHNIQUE}

This method is based on well known fact that in the area of impedance localization the tail particles of the passing bunch are experienced a transverse momentum kick. Value of the kick is proportional to the head particles shift from an axis and the total charge of the bunch. If a local bump of closed orbit is created just at the impedance location, then the orbit deviation appears while varying the beam current. Comparing two orbits measured for different currents we obtain the orbit deviation in the form of a betatron wave propagating from the bump location:

$$
\Delta y(s)=\frac{\Delta \Psi}{\sin \pi Q_{y}} \cdot \sqrt{\frac{\beta(s)}{\beta}} \cdot y_{0},
$$

where $y_{0}$ is the bump size, $\beta$ is average beta-function in the place of bunch localization. Amplitude of this wave yields information about reactive component of the transverse impedance. Note that the value of effect is:

$$
\Delta y_{\max } \cong \Delta \Psi \cdot y_{0} \text {. }
$$

The impedance measurement procedure is more complicated for purpose to avoid systematic errors, caused by current dependence and non-linearity of pickups. At first, the vertical and radial orbits are corrected to zero. The closed orbit is measured and stored at two current values: the maximum and the minimum. Then, the local vertical orbit distortion (bump) is produced using correctors on the measuring section (Figure 1) and orbit measurements are performed at two different current values close to those given above. Difference of these four orbits is the desired effect 
determined by an integral value of beta-weighted transverse impedance of the section at bump location.

The VEPP-4M is a mirror-symmetrical racetrack storage ring with the $366 \mathrm{~m}$ circumference. The ring consists of the technical straight section NT-ST; the halfrings SR, NR with the inserts SI, NI; and the experimental straight section SE-NE with mini-beta insert in the center. The azimuth zero is in the center of the technical straight section NT-ST.

A difference of two vertical orbits measured with and without a bump at the SI section of the VEPP-4M storage ring is shown in Figure 1. There are pickups data versus the VEPP-4M azimuth.

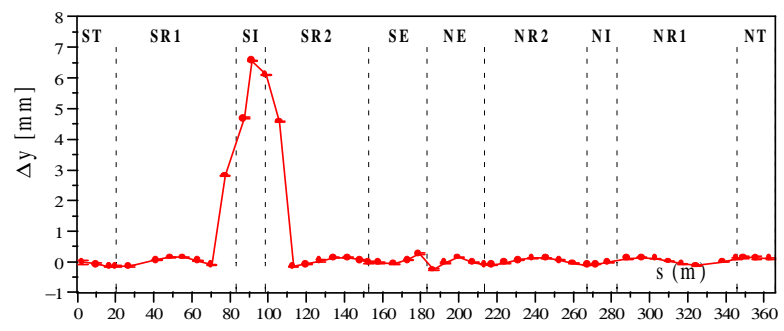

Fig. 1. Vertical orbit bump.

Figure 2 shows difference of four orbits in vertical and radial planes when the $6 \mathrm{~mm}$ vertical bump (Figure 1) was created. Section SI includes four vertical electrostatic separators and ten places of sharp change of the beam pipe cross section.

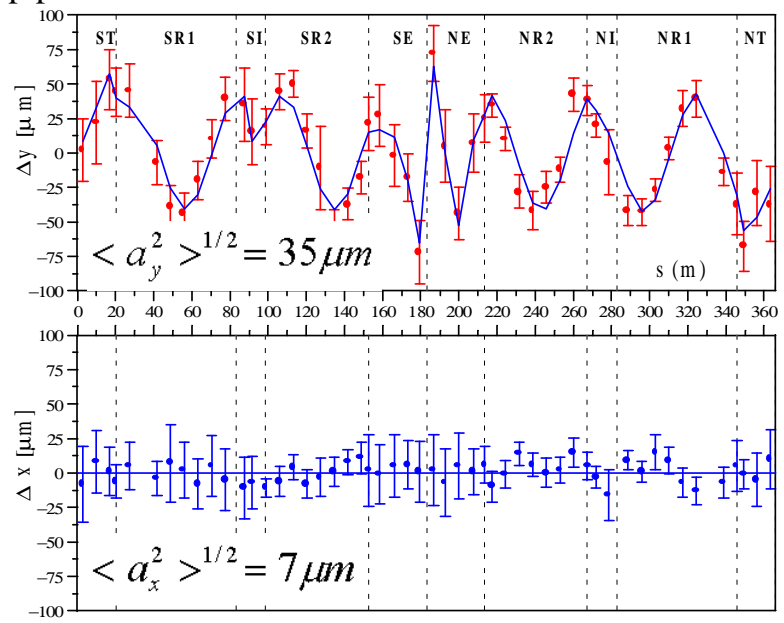

Fig. 2. Orbit deviation: measurement and simulation.

There are error bars in Figure 2, which sizes correspond to the measured values of pickups resolution $(\delta y)$. The results of numerical simulation are also shown in Figure 2 (solid line). This provides the possibility to determine the beta-weighted impedance value of SI section $<\operatorname{Im} Z_{y, 1} \cdot \beta_{y}>\cong 3.1 \pm 0.2 \mathrm{M} \Omega$. The value of the impedance in radial plane is within the error range. Accuracy of impedance determination in this method: $\frac{\Delta Z_{\perp}}{Z_{\perp}} \approx \frac{\delta y}{\left\langle a_{y}^{2}\right\rangle^{1 / 2}} \cdot \frac{1}{\sqrt{N_{\text {b.p.m. }}}}$, where $\left\langle a_{y}^{2}\right\rangle^{1 / 2}$ is the RMS amplitude of betatron wave (2), $N_{\text {b.p.m. }}$ is number of pickups along the ring. Distribution of transverse impedance along the VEPP-4M storage ring measured by the method described above is reported in work [3].

\section{METHOD VERIFICATION AND STUDY OF RESTRICTIONS}

Using this method we suppose that the main contribution into the closed orbit deviation is made by the dipole component of the transverse impedance. This assumption is valid for small bump values in axial-symmetrical structures. However, many of vacuum chamber elements are asymmetric and induced wake fields are in general nonlinear. To understand sphere of uses for this method and to proof correctness of measurement results treatment, we have carried out series of additional studies. Numerical calculation was made with program GdfidL for the section of vacuum chamber (Figure 3) in technical space. It contains the remote controlled vacuum-tight probe with luminescent screen, entered in aperture of beam pipe during of injection tuning. There are four such probes in technical section: NK1T, NK2T, SK1T and SK2T.
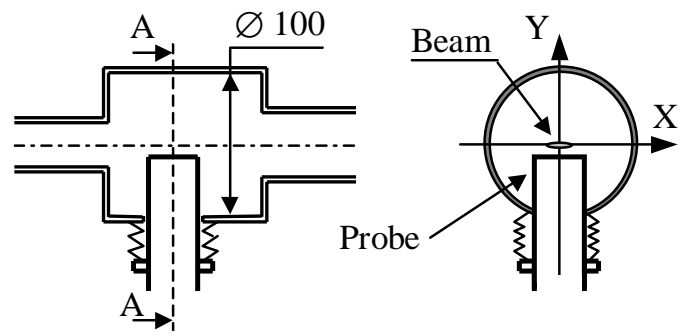

Fig. 3. The NK1T probe and the VEPP-4M beam pipe cross section (AA).

Distribution of $\mathrm{x}$-component of magnetic field (which deflects beam in $\mathrm{y}$-direction) along $\mathrm{y}$-direction is shown in Figure 4. This distribution corresponds to the moment when the bunch passes the probe center. Total charge of the bunch is $6 \mathrm{nC}$, RMS bunch length is $60 \mathrm{~mm}$.

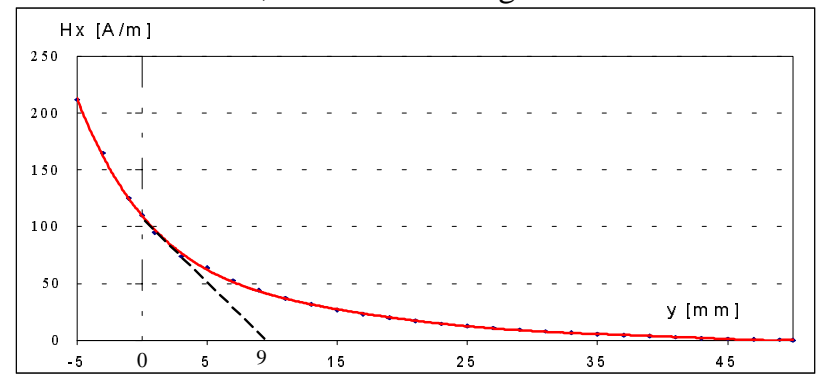

Fig. 4. Magnetic field distribution calculated using the code GdfidL. Probe is shifted at $-6 \mathrm{~mm}$ from axis.

Magnetic field is non-linear and assumption about main contribution of dipole component in the transverse impedance is no longer valid. If the bunch displacement from axis is small, we can linearize field about an axis and evaluate effective center of linear distribution. Distance from the bunch to the effective center 
( $y_{\text {eff }}=9 \mathrm{~mm}$, Figure 4 ) corresponds to the bump value for bump method mentioned above. This allows to determine effective dipole component of the impedance as transverse impedance normalized to the bunch displacement from the effective center. Presence of vacuum chamber elements which geometry can be changed, such as NK1T and other probes, allows to use another method of impedance measurement. In this case there is no bump created, but orbit deviation appears after probe pushing in.

Difference of two orbits measured with probe pushed in and out of beam pipe gives information about imaginary part of transverse impedance contributed by the probe in this structure:

$$
\Delta y(s)=\frac{I_{a} \cdot<\operatorname{Im}\left(\left(Z_{\perp}\right)_{2}-\left(Z_{\perp}\right)_{1}\right) \cdot \beta_{y}>}{2 \sqrt{2 \pi} \cdot(E / e) \cdot \sin \pi Q_{y}} \cdot \sqrt{\frac{\beta_{y}(s)}{\beta_{y}}},
$$

where $\left(Z_{\perp}\right)_{1}$ and $\left(Z_{\perp}\right)_{2}$ are integral values of impedance before and after of probe pushing, measured in $\Omega$.

Figure 5 shows such a difference for NK2T probe, pushed in beam pipe on $7.5 \mathrm{~mm}$ from pipe axis.

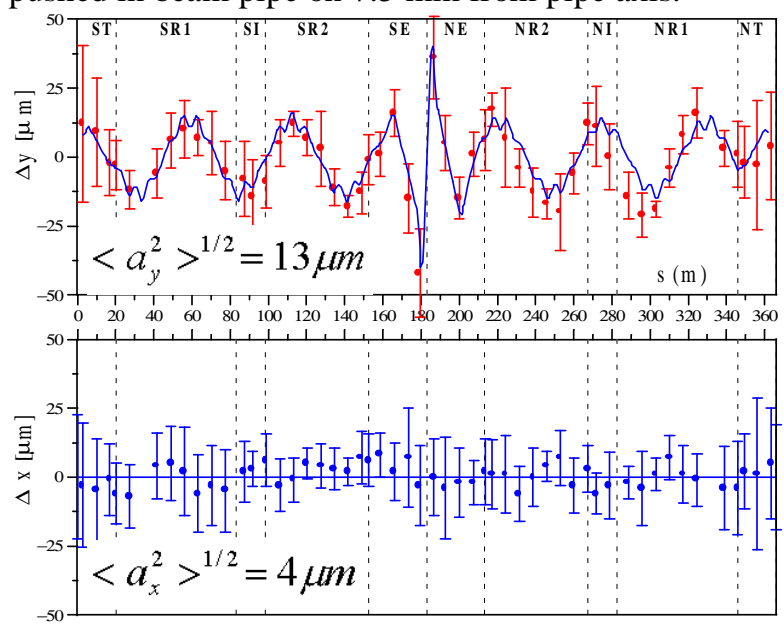

Fig. 5. Orbit deviation: measurements and simulations.

The results of numerical simulation are also shown in Figure 5 (solid line). Amplitude of vertical betatron wave corresponds to the impedance value of probe$$
<\operatorname{Im}\left(\left(Z_{\perp}\right)_{2}-\left(Z_{\perp}\right)_{1}\right) \cdot \beta_{y}>=6.2 \pm 1.4 \mathrm{k} \Omega \cdot \mathrm{m} .
$$

According to definition of the effective center of structure we can calculate effective dipole component of beta-weighted impedance which is $\left(Z_{\perp, 1}\right)_{\text {eff }}=Z_{\perp} / y_{\text {eff }}=0.69 \pm 0.15 \mathrm{M} \Omega$. The effect of probe on radial motion is small and lies in the error range (Figure 5b). Figure 6a shows vertical betatron wave with amplitude corresponding to sum of impedances of NK1T and NK2T probes. Distance between these probes is approximately one fourth of betatron oscillations wavelength, so effect is more than one probe by factor of two.

Figure $6 \mathrm{~b}$ shows the amplitude of the wave, corresponding to the impedance of NK2T and SK2T probes. Distance between these probes is approximately one half of betatron wavelength, so effect is localized in technical area and amplitude of wave in the ring is small and lies in the error range $\left(\left\langle a_{y}^{2}\right\rangle^{1 / 2}=5 \mu \mathrm{m}\right)$. In this case accuracy of method is decreased, because effect is measured by reduced number of pickups.

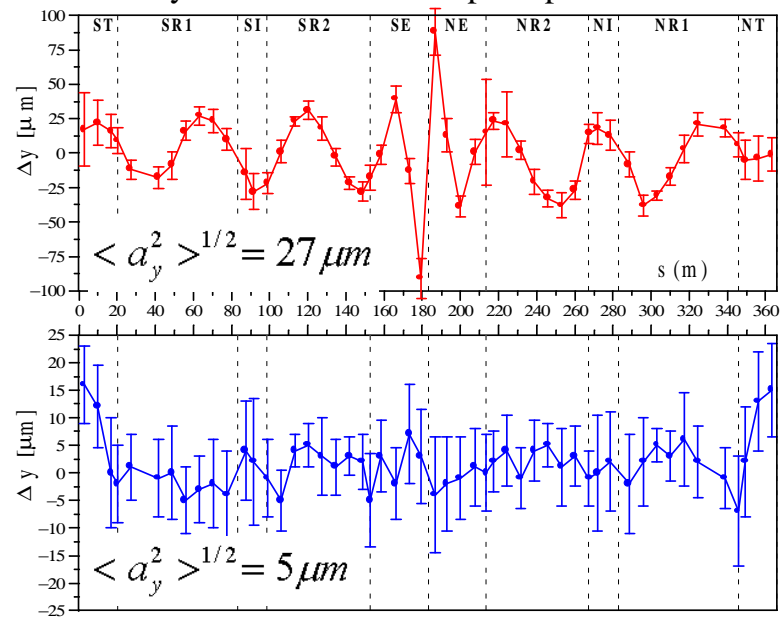

Figure 6. Orbit deviation: measurement and simulation.
a) Effect from NK1T and NK2T
b) Effect from NK2T and SK2T.

\section{CONCLUSION}

Verification and substantiation a new beam-based bump method for local transverse impedance measurement were made by the use of elements of vacuum chamber with the changing geometry. This method gives mainly a value of dipole component of the transverse impedance when small bumps are induced. It is verified by computer calculations with GdfidL. But in general this method provide measurement two-dimensional topology of wake field which acts on the bunch at the orbit bump location. Accuracy of this method is rather good because of local impedance evaluation is made by data, obtained from all pickups of the ring..

\section{ACKNOWLEDGMENT}

We wish to thank Warner Bruns for affording an opportunity to make calculations with GdfidL program.

\section{REFERENCES}

[1] V.Kiselev, V.Smaluk, "Experimental Study of Impedances and Instabilities at the VEPP-4M Storage Ring" // Proc. of EPAC'98

[2] J.Gareyte, J.S.Berg "Other Beam-Based Method to Measure Impedance" // Handbook of Accelerator Physics and Engineering, World Scientific Publishing, 1999

[3] V.Kiselev, V.Smaluk, "A Method for Measurement of Transverse Impedance Distribution along Storage Ring" // Proc. of DIPAC'99 